Volume: 1 | Volumen 1 | Número 1 | Number 1 | pp. 121 - 139 ISSN: 2634-355X (Print) | ISSN: 2634-3568 (Online) journals.tplondon.com/yeiya

First Submitted: 2 September 2020 Accepted: 3 December 2020 TRANSNATIONAL PRESS ${ }^{\circledR}$ DOI: https://doi.org/10.33182/y.v1i1.1166

\title{
Extractivismos, conflictos mineros y desarrollo desigual en América del Norte
}

\author{
Federico Guzmán López ${ }^{1}$
}

\section{Resumen}

Los extractivismos y la minería a gran escala en la región de América del Norte conformada por Canadá, Estados Unidos de América y México, se impulsaron en manos del capital privado transnacional, a partir del periodo neoliberal 1982-2020. Encontramos dos puntos de inflexión, el primero durante el año 1994 con la entrada en vigor del Tratado de Libre Comercio de América del Norte (Tlcan) y el segundo durante el año 2020 con la renegociación comercial mediante el Tratado entre México, Estados Unidos y Canadá (T-MEC) y la pandemia global del COVID-19. Los resultados registran siete tendencias: incrementos de conflictos mineros, de precios internacionales y reservas del oro en bancos centrales, relación desigual capital-trabajo, fortalecimiento de China en minería, continuar operaciones durante pandemia y Responsabilidad Social Empresarial.

Palabras clave: Canadá; Estados Unidos de América; megaminería; México; tratados comerciales

\begin{abstract}
Extractivism, mining conflicts, and uneven development in North America

Extractivisms and large-scale mining in the North American region made up of Canada, the United States of America and Mexico, were promoted in the hands of transnational private capital, from the neoliberal period 1982-2020. We found two turning points, the first during 1994 with the entry into force of the North American Free Trade Agreement (NAFTA) and the second during 2020 with the trade renegotiation through the Agreement between Mexico, the United States and Canada. (T-MEC) and the global pandemic of COVID-19. The results register seven trends: increases in mining conflicts, international prices and gold reserves in central banks, unequal capital-labor ratio, strengthening of China in mining, continuing operations during the pandemic and Corporate Social Responsibility.
\end{abstract}

Keywords: Canada; United States of America; mega-mining; Mexico; trade agreements

\section{Introducción}

En la región de América del Norte la dinámica del modelo neoliberal hegemónico durante el periodo 1982-2020, permitió que los extractivismos y sus consecuencias expresadas en conflictos socioambientales y mineros, en conjunto contribuyeron a que se acentuara el desarrollo desigual entre Canadá, Estados Unidos de América y México. Se trata de un problema agrario de justicia ambiental que día con día afecta a más habitantes, el cual ha sido poco estudiado de forma integral a nivel regional.

A partir de los datos oficiales que brindan los gobiernos de los países que conforman la región de América del Norte, así como de otras fuentes de organismos públicos y privados del ámbito

\footnotetext{
${ }^{1}$ Universidad Autónoma de Zacatecas. Zacatecas, México. Correo electrónico: federic7bcm@gmail.com
} 
internacional, encontramos que en conjunto Canadá, Estados Unidos de América y México conforman un territorio de 21.8 millones de kilómetros cuadrados de superficie para el año 2018, cifra equivalente al 16.5 por ciento de los 132.02 millones de kilómetros cuadrados de la superficie territorial de todos los países del mundo (Banco Mundial, 2020). Está región contaba con una población de 493.40 millones de habitantes para el año 2019, equivalente al 6.4 por ciento de los 7,674 millones de habitantes a nivel mundial (Banco Mundial, 2019).

Por su parte, al revisar la lista de los veinte principales países productores de oro a nivel mundial, según el Consejo Mundial del Oro (2020a), Estados Unidos de América se ubicó en cuarto lugar, Canadá en quinto lugar y México en noveno lugar mundial, respectivamente. En conjunto las tres naciones mencionadas produjeron 494.5 toneladas de oro durante el año 2019, equivalente al 15.8 por ciento de la producción total del mineral aurífero mundial que alcanzó las 3,272.7 toneladas producidas por 43 de los 193 países del mundo reconocidos por las Naciones Unidas oficialmente (Naciones Unidas, 2020).

En cuanto a la lista de los 20 principales países productores de plata a nivel mundial para el año 2019, de acuerdo con The Silver Institute (2020), México se ubicó en el primer lugar, Estados Unidos de América en el lugar número diez y Canadá en el lugar número 14 a nivel mundial, respectivamente. En conjunto los tres países señalados produjeron 235.3 millones de onzas del mineral argentífero, equivalente al 28.1 por ciento del total producido a escala mundial que alcanzó los 836.5 millones de onzas.

En cuanto al índice de atracción de inversiones según el Fraser Institute (2019: 4-6, 10-11), los datos de la encuesta anual a empresas mineras 2019, aplicada a 2,400 empresas alrededor del mundo, mismas que reportaron montos de inversión en exploración minera por USD \$1.8 mil millones de dólares durante el año 2018 y USD \$1.9 mil millones de dólares, durante el año 2019, respectivamente. En el año 2018 los países y provincias de América del Norte se ubicaron en los primeros lugares a nivel mundial, de Canadá: Saskatchewan 3/83, Quebec 4/83 y Yukón 9/83 lugar; en Estados Unidos de América: Nevada 1/83, Alaska 5/83 y Utah 7/83, mientras que México se ubicó en el lugar 29/83. Para el año 2019, en Estados Unidos de América: Nevada 3/76, Alaska 4/76, Idaho 8/76 lugar a nivel mundial; en Canadá: Saskatchewan 11/76, Ontario 16/76 y Quebec 18/76 lugar mundial, por su parte México se ubico en el lugar mundial 38/76.

Tan solo la distribución geográfica de los activos de la minería canadiense en la región de América del Norte durante el año 2017, de acuerdo con The Mining Association of Canada (2019: 75), en Canadá un total de 1,021 empresas sumaron USD \$91.4 billones de dólares, en Estados Unidos de América 286 empresas contaban con $\$ 24.9$ billones de dólares y en México 110 compañías transnacionales de megaminería de origen canadiense contaban con USD \$18.4 billones de dólares.

Asimismo, en el caso de México, de acuerdo con la Secretaría de Economía, (2020), además de ubicarse en el quinto lugar mundial en materia de atracción de inversiones y exploración minera, durante el año 2018 la minería recibió inversiones por la cantidad de USD \$4,897 millones de dólares. En contraste con esos montos de inversión de acuerdo con Eme Equis (2020), la Cámara Minera de México (Camimex), reportó que el sector minero en el país ha sufrido estancamiento, como consecuencia del entorno económico de incertidumbre, que han propiciado el escenario político derivado de las decisiones y políticas públicas del Gobierno de México durante los dos primeros años de la presidencia en manos de Andrés Manuel 
López Obrador, principalmente por la pérdida de estabilidad política, desaparición de la Subsecretaría de Minería de la estructura orgánica de la Secretaría de Economía, disminución de atracción de inversiones, aumento de proyectos suspendidos o diferidos. Lo que trajo consigo una disminución del 25 por ciento en inversión para exploración minera y que 523 megaproyectos de minería se encontraban detenidos durante el año 2019.

A partir de los datos anteriores se plantea la siguiente pregunta, ¿Cómo y de qué manera se manifiesta la dinámica de los extractivismos y la minería a gran escala en la región de América del Norte y sus consecuencias socioambientales?

El objetivo del presente artículo es revisar la transición del extractivismo minero en la región de América del Norte durante el periodo neoliberal, con énfasis en el contexto de los tratados comerciales, las crisis de 2008 y 2020, así como la pandemia global del COVID-19.

Se argumenta que la minería a gran escala que predomina en los países de la región de América del Norte, ha significado una dinámica de desarrollo regional desigual en las esferas de la producción y el mercado de minerales metálicos, cuyos principales beneficiados de la renta minera han sido las empresas transnacionales y gobiernos de Canadá y Estados Unidos de América, en contraste el más perjudicado ha sido México, configurado en territorio de sacrificio, con mayor agotamiento de los recursos naturales y afectaciones eco-sociales expresadas en mayores conflictos mineros.

El artículo se dividió en tres apartados, en el primero se presenta un esbozo teórico metodológico. En el segundo se ofrece un mapeo de los conflictos mineros en la región de América del Norte en el contexto mundial, tomando en cuenta la intensidad de los conflictos, la distribución geográfica y los impactos ambientales.

En el tercero se abordan las siete tendencias de la minería a gran escala en el contexto de la crisis financiera y la pandemia del COVID-19. Al final se presentan las conclusiones sobre la realidad y desafíos de otra forma de explotar minerales.

\section{Esbozo teórico metodológico sobre extractivismos, conflictos mineros y desarrollo desigual}

Para adentrarnos en el estudio sobre los extractivismos, conflictos mineros y desarrollo desigual, se consideró la ecología política, que ayuda a explicar la dinámica de la génesis y efectos de los conflictos de justicia ambiental (Martínez, 2005), acompañados del desarrollo desigual regional y entre naciones del norte y el sur global (Robbins, 2004). Según Francisco (2020: 2), en la reciente encíclica destacó la ausencia de fraternidad: "los conflictos locales y el desinterés por el bien común son instrumentalizados por la economía global”.

Cuando hablamos en singular del concepto extractivismo, según Gudynas (2015: 9, 14), "se refiere a la apropiación de recursos naturales para exportarlos". Para comprender ese término expresado en plural como extractivismos, de acuerdo con dicho autor, se refiere a grandes emprendimientos llevados a cabo en las explotaciones minera (megaminería a cielo abierto, en más de un mil hectáreas de superficie de tierras y remueven por año más de un millón de toneladas de roca), petrolera y en el monocultivo destinado a la producción de alimentos destinados para el mercado exterior, que ha significado el acaparamiento de grandes extensiones de tierra, para extraer grandes volúmenes de recursos naturales, principalmente minerales, energéticos, alimentos y agua proveniente de fuentes superficiales y subterráneas, 
llevado a cabo en mayor medida por empresas de capital privado transnacional y así como por Estados nacionales (Gudynas, 2015: 7).

Aunque debemos tomar en cuenta que históricamente hemos tenido viejos extractivismos, como la dinámica de economía de enclave referida a la explotación de minerales metálicos, principalmente que se transferían desde la época de la colonización de Latinoamérica para proveer a Europa, principalmente España e Inglaterra, con bajo impacto positivo en la economía local (LeGrand, 2006 y Turdera, 2012).

Mientras que los nuevos extractivismos se alentaron por el auge de los precios internacionales de las materias primas, lo que motivó a que los gobiernos de varios países centraran sus proyectos de desarrollo nacional en mecanismos de carácter extractivista y de manera más reciente neoextractivista, para acceder a una mayor porción de la renta minera y petrolera, para asignar recursos a programas sociales de combate a la pobreza, así como por los supuestos beneficios económicos en materia de empleo e inversión productiva.

De acuerdo con la propuesta de Gudynas (2015: 22-26), de cuatro generaciones de extractivismos en la minería considerando la intensidad de los recursos naturales apropiados, la primera generación se refería a la minería de socavón, basada en la fuerza humana y tracción animal. La segunda generación, con el uso de explosivos, máquinas de combustión interna o de vapor a partir de la mitad del siglo XIX. La tercera generación se refiere a la megaminería a cielo abierto desde finales de la década de 1970, con uso de excavadoras, camiones de carga pesada, separación de minerales, grandes volúmenes de residuos y de consumo de agua. La cuarta generación durante los inicios del siglo XXI con la minería submarina, que se pretende llevar a cabo en casos como el proyecto Don Diego, por parte de la empresa transnacional estadounidense Odyssey Marine Exploration, en Baja California Sur, desde el año 2015 (Centro de Información sobre Empresas y Derechos Humanos, 2015).

Sobre los elementos metodológicos se consideró como base de información empírica el Atlas de Justicia Ambiental (Ejatlas), que permite la conexión teórico-metodológica de la ecología política con las resistencias protagonizadas por los movimientos sociales que soportan sus acciones mediante colectivos de base comunitaria. Porque es la principal herramienta de análisis cualitativa, cuantitativa y con datos georreferenciados de los 3,255 conflictos socioambientales que existen en 167 países alrededor del mundo, así como los 637 conflictos mineros que se han registrado actualmente en 91 países (Ejatlas, 2020).

\section{Mapeo de conflictos mineros en América del Norte}

Los conflictos socioambientales y mineros en América del Norte y otras regiones del mundo surgieron en un contexto global marcado principalmente por tres dinámicas de alcance globallocal, en primera instancia la instrumentación del modelo neoliberal desde la década de 1980, posteriormente por las reformas estructurales promovidas durante la década de 1990 para favorecer la privatización de los recursos naturales, recientemente derivado de las crisis financieras que detonaron durante las dos primeras décadas del siglo XXI.

Según el Ejatlas (2020) de los 3,255 conflictos de justicia ambiental registrados al 30 de septiembre de 2020, de los cuales, al revisar los datos de los países ubicados en los doce primeros lugares a nivel mundial, encontramos que Estados Unidos de América se ubicó en el cuarto, México en el sexto y Canadá en el décimo segundo lugar, respectivamente (ver figura 1). 
Figura 1. Número de conflictos socioambientales en los países ubicados en los 12 primeros lugares a nivel mundial, 2020.

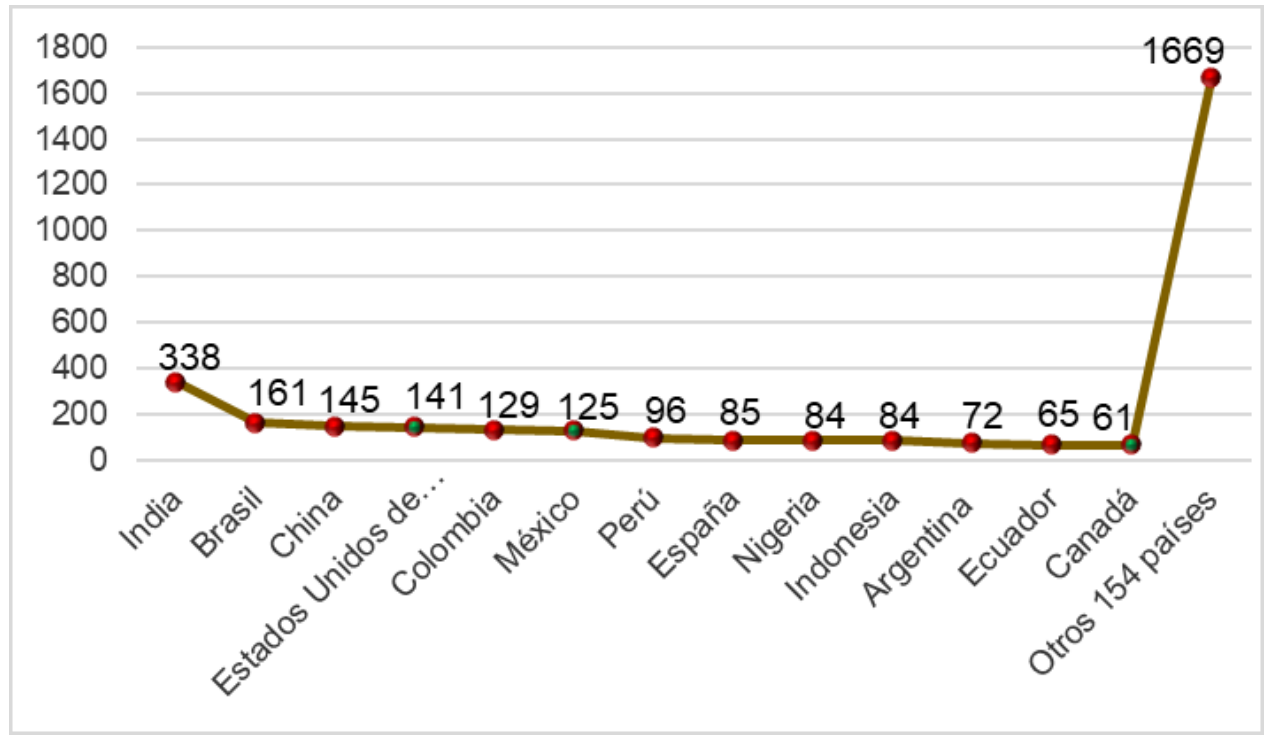

Fuente: Ejatlas (2020)

Los datos de la figura anterior indican que, tan solo 13 países concentraron 1,586 conflictos socioambientales, equivalentes al 48.7 por ciento del total mundial, de los cuales, 327 correspondieron a los países de la región de América del Norte: Estados Unidos de América (141), México (125) y Canadá (61), cifra equivalente al 10 por ciento del total de conflictos a escala mundial. Además, se menciona que Nigeria e Indonesia registraron un empate de 84 conflictos socioambientales cada uno, ubicándose ambos en el noveno lugar mundial, (ver figura 2).

Figura 2. Mapa de conflictos socioambientales en la región de América del Norte, 2020.


Fuente: Ejatlas (2020) 
Asimismo, al revisar la distribución de los 327 conflictos socioambientales, clasificados en las diez categorías que aparecen en el Ejatlas, observamos el siguiente comportamiento, (ver figura 3).

Figura 3. Porcentaje de conflictos socioambientales en América del Norte agrupados en diez categorías, 2020.

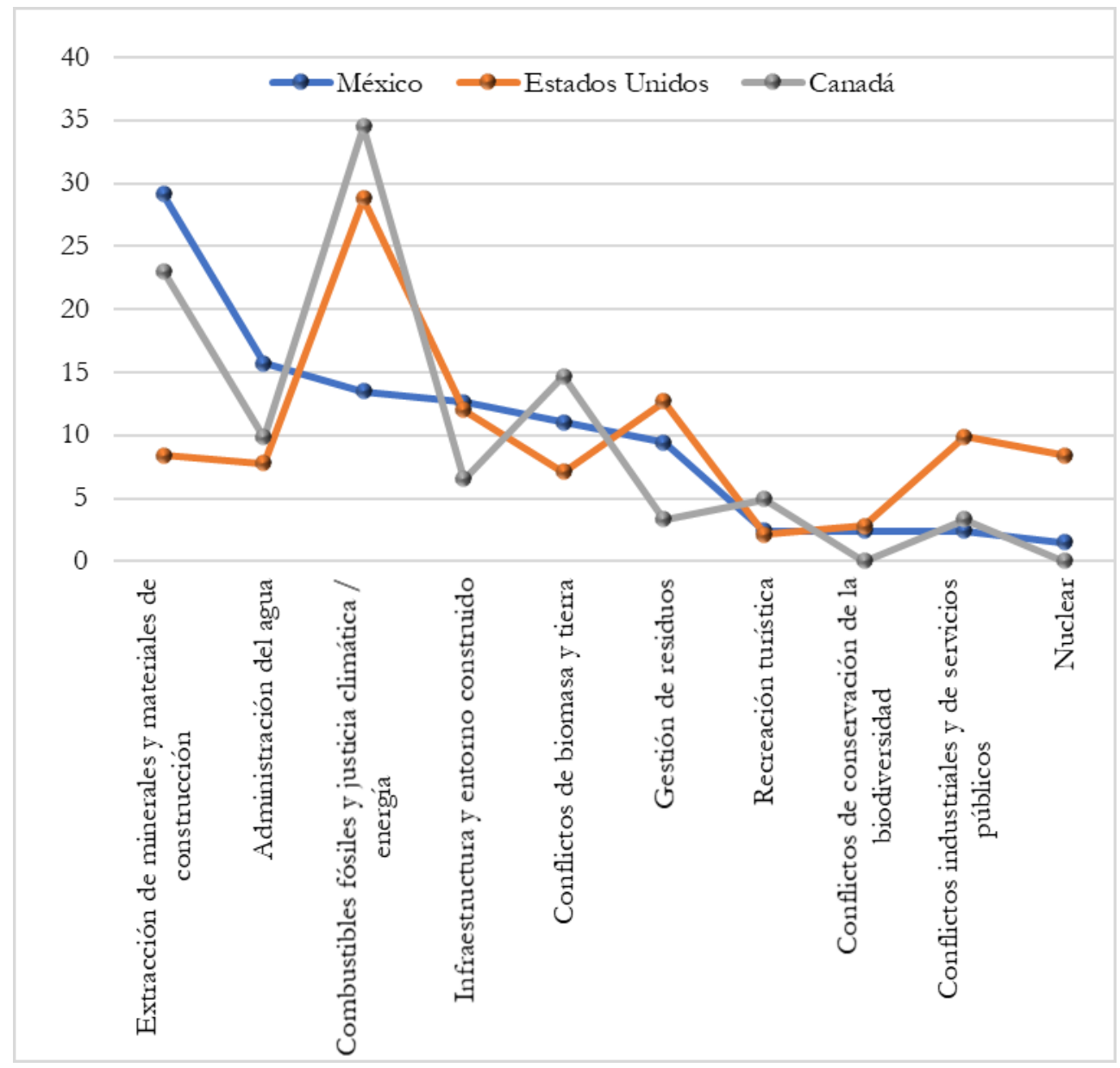

Fuente: Ejatlas (2020)

Los datos de la figura anterior indican que, de las diez categorías, el relacionado a la minería en México alcanzó los 37 casos y se ubica en el primer lugar con el 29.1 por ciento de los 125 conflictos socioambientales de dicho país; en Canadá la minería alcanzó los 14 casos, ubicado en el segundo lugar con el 23 por ciento de los 61 casos de los conflictos socioambientales en dicha nación y Estados Unidos de América registró 12 casos de minería, ubicado en el quinto lugar con el 8.4 por ciento de los 141 conflictos socioambientales de dicho país. Por tanto, de los tres países de América del Norte, México es el territorio más sacrificado y afectado por la mayor presencia de conflictos mineros en términos absolutos y relativos.

Por su parte, al revisar la distribución geográfica de los 637 conflictos mineros del mundo, estos últimos en su mayoría se localizan en los 18 países ubicados en los primeros doce lugares 
a nivel mundial, de los cuales seis lugares cuentan con dos países con el mismo número de conflictos (ver figura 4).

Figura 4. Número de conflictos mineros en los países ubicados en los 12 primeros lugares a nivel mundial, 2020.

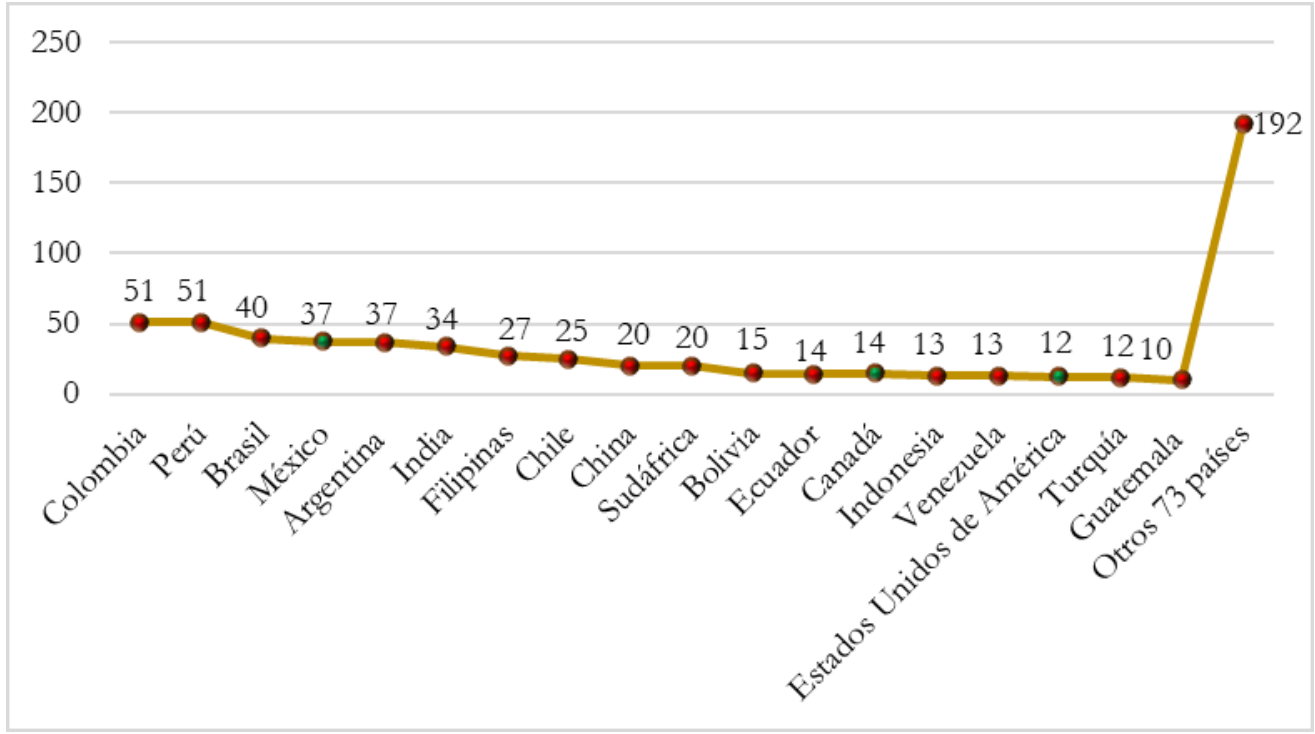

Fuente: Ejatlas (2020).

Los datos de la figura anterior indican que tan solo 18 países concentran el 69.8 por ciento de los conflictos mineros, además de las naciones que conforman América del Norte en conjunto cuentan con 63 casos, equivalente al 9.9 por ciento del total de los casos registrados a escala global. Además, México se ubicó en el tercer lugar mundial con 37 casos, Canadá en el noveno lugar con 14 casos y Estados Unidos de América en el décimo primer lugar con 12 casos. Es decir, México tiene un 42.3 por ciento más de conflictos mineros que Canadá y Estados Unidos de América conjuntamente.

\section{Intensidad de los conflictos mineros en los países de América del Norte}

En el Ejatlas los conflictos se agrupan en intensidades alta, media, baja y corresponde a los grados de violencia y de movilización de los grupos participantes. Asimismo, los conflictos latentes, se refiere a los que aún no se manifiestan socialmente. De los 63 casos de conflictos mineros por grado de intensidad en la región de América del Norte, se obtuvo lo siguiente (ver figura 5).

Las cifras de la figura anterior, señalan que en México predominan los conflictos mineros de intensidad alta con un 43 por ciento del total, en contraste los casos de Canadá y Estados Unidos de América apenas alcanzan el 7 y 17 por ciento, respectivamente, ambos según Roy (2018) estuvieron muy por debajo del promedio mundial de conflictos de alta intensidad que registró el 29.2 por ciento del total. Lo que indica que México es el país de la región de América del Norte donde además de destacar en términos absolutos por el mayor número de conflictos mineros, también sobresale porque estos se llevan a cabo con mayor grado de violencia, represión policiaca y más intensivos niveles de movilización masiva, detenciones, y además la 
pérdida de vidas humanas por asesinato. Como ocurrió en cinco conflictos mineros, donde al menos a 12 personas les quitaron la vida, como los casos emblemáticos de los campesinos Bernardo Vásquez en San José del Progreso, Oaxaca y Mariano Abarca en Chicomuselo, Chiapas. Mientras que en Estados Unidos de América y Canadá el Ejatlas, no registró ningún caso de asesinato a personas de las comunidades que se resisten a los megaproyectos mineros.

Figura 5. Grado de intensidad de los conflictos mineros en América del Norte, 2020 (porcentaje).

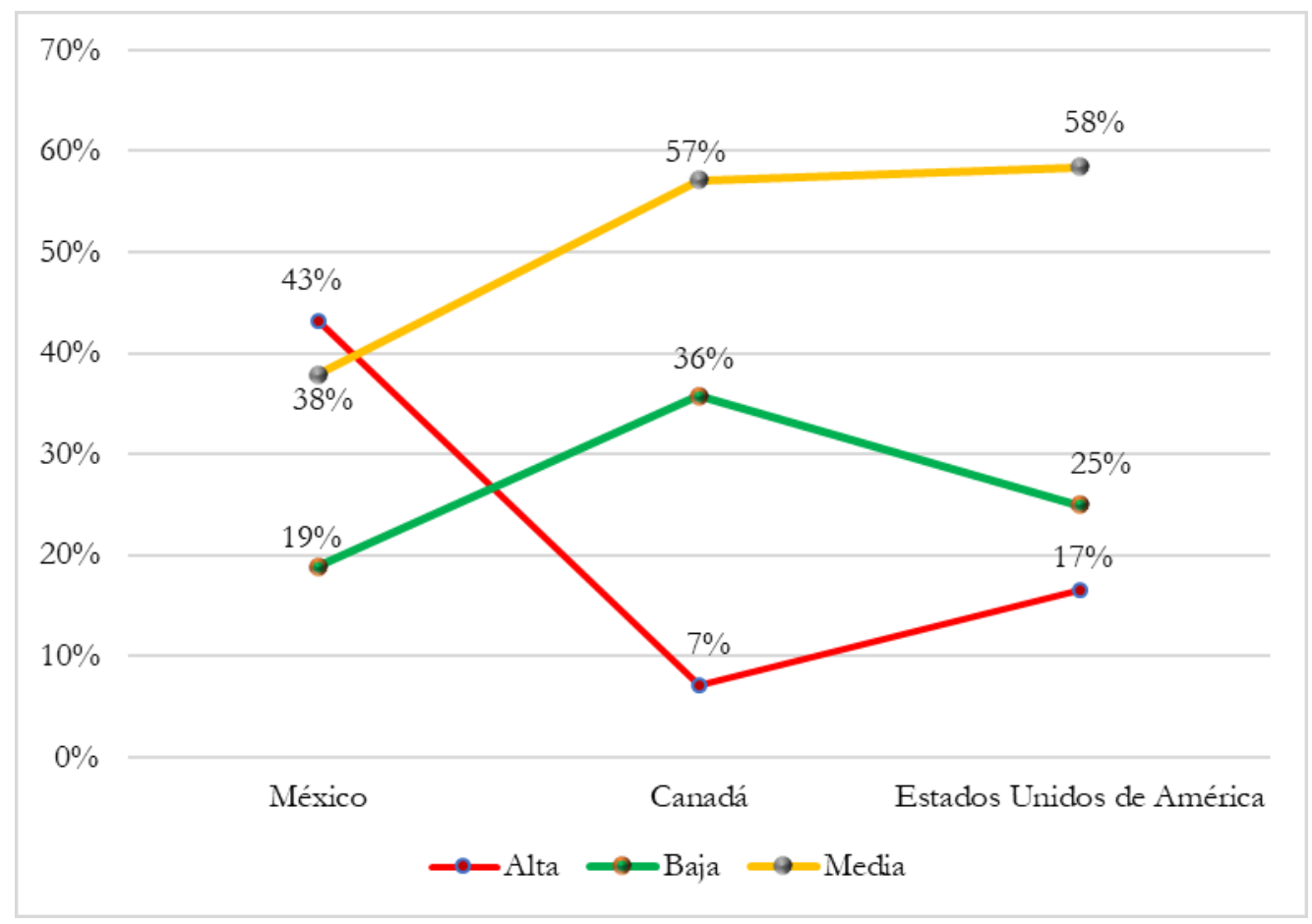

Fuente: Ejatlas (2020).

\section{Distribución geográfica de los conflictos mineros}

Un signo distintivo de la ampliación de las fronteras del extractivismo minero en las naciones de la región de América del Norte es que tanto en Canadá, Estados Unidos de América y México durante los primeros años del siglo XXI se empezaron a visibilizar los primeros casos de conflictos mineros por afectaciones a población semiurbana y urbana, aunque en mayor proporción en el territorio mexicano (ver figura 6).

Los datos de la figura anterior, indican que en mayor medida los conflictos mineros en América del Norte han surgido en territorios de áreas rurales con población campesina e indígena, aunque gradualmente las comunidades afectadas por la minería también empiezan a brotar en zonas semiurbanas y urbanas.

Además de lo antes señalado, al revisar los casos en que derivado de las acciones colectivas de resistencia para parar temporal o definitivamente algún megaproyecto minero encontramos que en Canadá el 25 por ciento de los casos son considerados éxito de justicia ambiental, el 18.9 por ciento en México y el 7.1 por ciento en Estados Unidos de América. Tal situación 
indica que Canadá que representa el imperialismo minero hegemónico a nivel global, en su territorio se tienen los mayores datos de éxito de justicia ambiental, cuando los pueblos indígenas y otras personas que viven y trabajan en zonas rurales, salen en defensa de su territorio y recursos naturales, han logrado que los gobiernos nacionales y locales se encarguen de salvaguardar de mejor manera sus derechos humanos (Ejatlas, 2020).

Figura 6. Distribución geográfica de los conflictos mineros en América del Norte, 2020 (porcentaje).

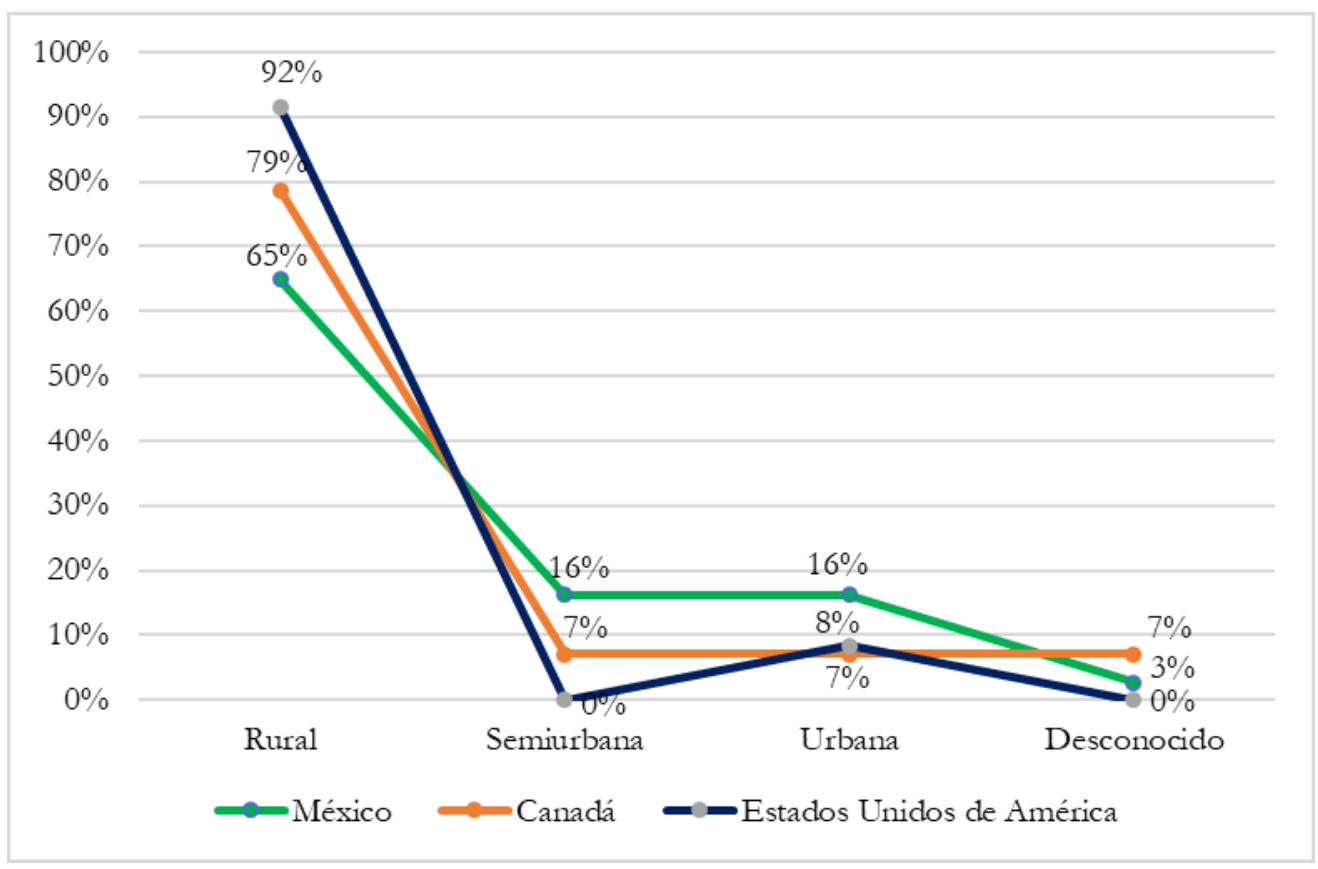

Fuente: Ejatlas (2020).

\section{Impactos ambientales de los conflictos mineros en América del Norte}

La apertura comercial se acentuó con la firma del Tlcan en el año de 1994, se intensificó el volumen de inversión de capital privado en la megaminería y por consecuencia, se agudizaron las devastaciones de los recursos naturales. Los 63 casos de conflictos mineros que han surgido en la región de América del Norte, tuvieron los impactos ambientales siguientes (ver tabla 1).

La información de la tabla 1, indica que, de los siete rubros de impactos ambientales relacionados a los conflictos mineros en América del Norte, acumulados al año 2020, a nivel general los rubros de contaminación del agua y destrucción de biodiversidad, acumularon el 47.5 por ciento del total de los casos registrados de impactos en dicha región. Sin embargo, cuando vemos la situación de los impactos ambientales de la minería específicamente en cada país, en el caso de México la contaminación del suelo y el agua registraron el 51.7 por ciento del total de los impactos en dicho país. Por su parte en Canadá el 57.1 por ciento de los impactos se concentró en los rubros de destrucción de biodiversidad y desastre de desechos mineros, mientras que, en Estados Unidos de América, fue la contaminación del agua y la 
destrucción de los bosques que alcanzó el 50 por ciento de los impactos ambientales desfavorables por la megaminería.

Tabla 1. Inventario de impactos ambientales relacionados con los conflictos mineros de América del Norte, (2020).

\begin{tabular}{lccc}
\hline \multicolumn{1}{c}{ Impacto ambiental } & México & Canadá & $\begin{array}{c}\text { Estados Unidos de } \\
\text { América }\end{array}$ \\
\hline Contaminación de fuentes de agua & 32 & 10 & 12 \\
\hline Contaminación del suelo & 29 & 2 & 1 \\
\hline Destrucción de biodiversidad & 21 & 13 & 10 \\
\hline Pérdida de seguridad alimentaria & 12 & 2 & 1 \\
\hline Contaminación del aire & 12 & 2 & 10 \\
\hline Desastre de desechos mineros & 9 & 11 & 11 \\
\hline Destrucción de bosques y paisaje & 3 & 2 & \\
\hline
\end{tabular}

Fuente: Ejatlas (2020-9.

\section{Siete tendencias de la minería a gran escala en el marco de la crisis financiera de 2020 y el COVID-19}

\section{1.- Incremento de precios del oro}

$\mathrm{Al}$ inicio del periodo neoliberal los precios internacionales del oro según el Consejo Mundial del Oro (2020b), registraron una cotización internacional de USD $\$ 395.00$ dólares por onza el día 3 de enero de 1982. Durante el periodo 1982-2020, en términos generales registraron una tendencia creciente, con fases cortas de inestabilidad y dos puntos de inflexión relacionados con las crisis económicas registradas durante los años 2008 y 2020, hasta alcanzar dos niveles de precios máximos históricos, el primero de USD \$1,895 dólares por onza de oro alcanzado el 5 de septiembre de 2011 y luego el segundo por un monto de USD\$2,067.15 dólares por onza de oro el 6 de agosto de 2020 (ver figura 7).

Los datos de la figura anterior indican que, al revisar el comportamiento de los precios internacionales del oro durante las dos últimas décadas del siglo XX, registraron una caída del 35 por ciento, al pasar de USD\$395.00 dólares por onza de oro durante 1982 hasta descender a los USD\$256.5 dólares por onza en el año de 1999.

En contraste durante las dos primeras décadas del siglo XXI, los precios internacionales del oro tuvieron una tendencia creciente, porque para el año 2000 registraron una cotización de 279.40 dólares por onza, hasta conseguir los USD \$2,067.15 dólares por onza de oro el 6 de agosto de 2020, equivalente a un incremento del 639.8 por ciento durante el periodo 20002020. Esta volatilidad pronunciada durante las dos primeras décadas del siglo XXI, se debió inicialmente a la tensión en las bolsas de valores a consecuencia del atentado a las torres gemelas de la ciudad de Nueva York, Estados Unidos de América y posterior por las crisis de 2008 y 2020.

Otro elemento a resaltar es que en el marco de la crisis financiera desatada por la emergencia sanitaria por la pandemia del COVID-19, las cotizaciones internacionales del oro crecieron un 35.4 por ciento durante los nueve primeros meses del año 2020, debido a que 1 de enero de 2020 era de USD $\$ 1,527.1$ dólares por onza y aumentaron hasta los USD \$2,067.15 dólares por onza de oro el 6 de agosto de 2020, con una ligera variación negativa hasta colocarse en los USD\$1,886.90 el día 30 de septiembre de 2020 (Consejo Mundial del Oro, 2020b). 
Figura 7. Cotizaciones internacionales del oro, 1982-2020 (dólares por onza).

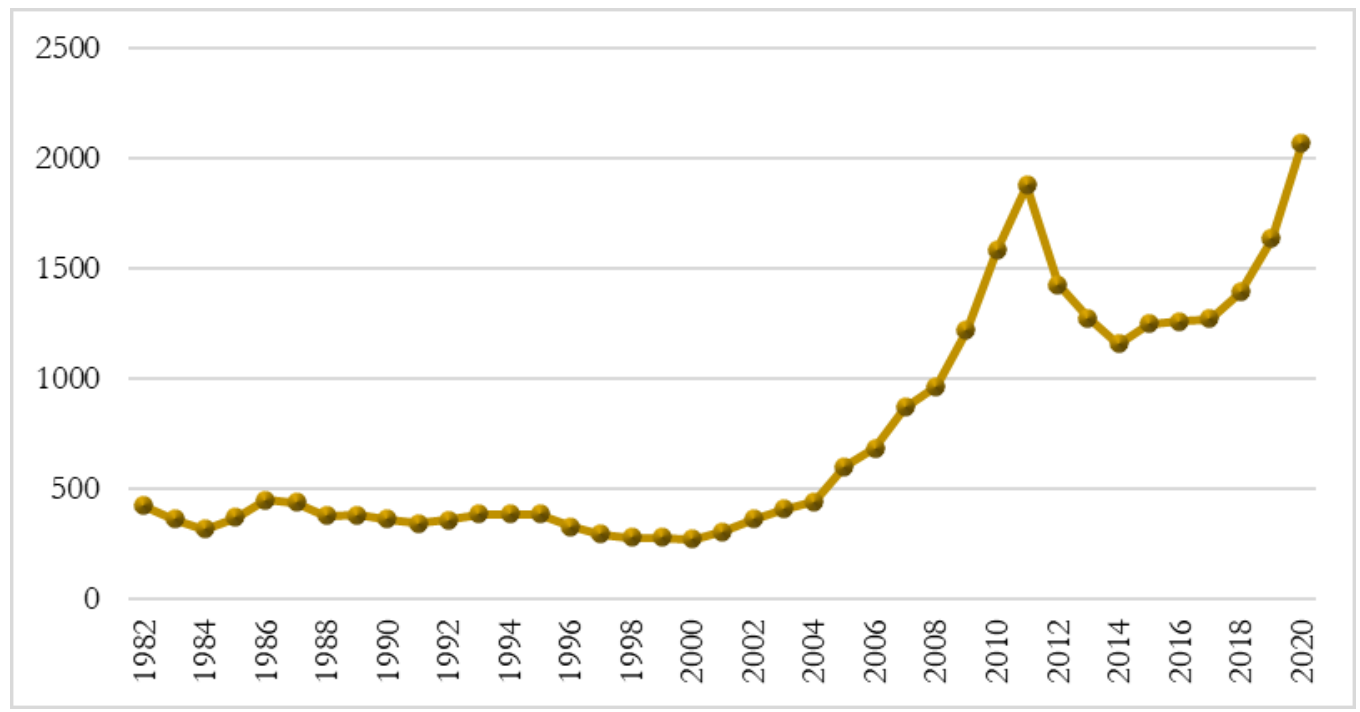

Fuente: Basado en Consejo Mundial del Oro (2020b). La cotización del oro del año 2020 corresponde a los precios internacionales registrados con el máximo histórico el día 6 de agosto de 2020.

\section{2.- Incremento de reservas de oro en bancos centrales}

Durante las crisis financieras ocurridas en los años 2008 y 2020, se registró una tendencia general de incremento absoluto y relativo de la oferta y demanda global de oro en los bancos centrales, debido a que durante el año 2010 fue de 79.2 toneladas, equivalente al 1.9 por ciento del total de la oferta y demanda mundial de oro que ascendió a las 4,185 toneladas. Mientras que para el año 2019 alcanzó las 648.2 toneladas de metal aurífero, equivalente al 14.8 por ciento de las 4,368.3 toneladas de la oferta y demanda mundial de oro, de las cuales el resto fueron 2,118.6 toneladas (48.5 por ciento) para joyería, 1,274.9 toneladas (29.2 por ciento) para inversión en lingotes y monedas, 326.6 toneladas (7.5 por ciento) para tecnología (Consejo Mundial del Oro, 2020c).

En cuanto al volumen de reservas de oro en bancos centrales durante el periodo de 20002020, se registró una propensión de incremento desde las 33,443.6 toneladas del metal precioso a inicios del año 2000 y creció hasta alcanzar las 34,891.5 toneladas de oro durante el año 2020, cifra equivalente al 4.3 por ciento de incremento acumulado en el periodo señalado.

En el caso de la región de América del Norte, las reservas de oro durante el primer trimestre del año 2000 fueron de 46.2 toneladas en Canadá, 6.8 toneladas en México y de 8,138.8 toneladas en Estados Unidos de América; en conjunto los tres países acumularon el 24.5 por ciento del total de reservas de oro a nivel mundial y durante el segundo trimestre de 2020 llegaron a cero toneladas en Canadá, 120 toneladas en México y 8,133.4 toneladas en Estados Unidos de América, es decir concentraron el 23.6 por ciento de las reservas de oro a escala global. En este rubro México registró una variación positiva en el volumen de reservas, mientras que Canadá y Estados Unidos de América tuvieron variación negativa (Consejo Mundial del Oro, 2020c). 


\section{3.- Relación desigual capital-trabajo en minería en América del Norte}

En América del Norte el contexto de la transición del Tlcan al T-MEC, no ha significado en los hechos una mejora inmediata a las asimetrías salariales que existen entre los trabajadores mineros de los tres países de la región, porque, mientras en México un trabajador del sector minero recibe un salario de USD $\$ 20$ dólares en promedio por jornada laboral diaria, en cambio los trabajadores mineros de Canadá y Estados Unidos de América perciben en promedio USD $\$ 40$ dólares por hora. Es decir, que los trabajadores mineros de Canadá y Estados Unidos de América reciben un 1,500 por ciento más de salario por jornada diaria que los mineros mexicanos (Navarro, 2014).

Por su parte al revisar las estadísticas de empleos en el sector minero en la región de América del Norte, en el caso de Canadá durante el año 2018 alcanzó los 626,000 empleos, de los cuales fueron 409,000 personas empleadas directamente y 217,000 empleos indirectos (The Mining Association of Canada, 2019: 45). En Estados Unidos de América la cifra de personas ocupadas en las actividades económicas del sector minero para el año 2018 alcanzó las 654,000 personas (Oficina de Estadísticas Laborales de EE.UU., 2018). Para el caso de México, el sector minero del país únicamente generó empleos para 379,020 personas durante el año 2018, (Servicio Geológico Mexicano, 2019: 17), por tanto, fue el que menor cantidad de empleos generó en el sector minero en la región de América del Norte.

\section{4.- Fortalecimiento de China en la cadena de valor global de la minería a gran escala.}

Existen al menos tres signos vitales de que China se perfila a disputar el imperialismo minero canadiense y salir victoriosa a partir de la tercera década del siglo XXI, lo que le permitirá el control de la cadena de valor global de la minería. Principalmente porque durante el año 2020 se intensificó la compra de activos de empresas canadienses por parte de empresarios chinos (El Economista, 2 de diciembre de 2019), además tiene mayor representación en el mercado

de los minerales de litio y tierras raras, que son la base para las energías renovables (Bejerano, 2019). Asimismo, según Lampadia (2016) Shanghai, China también le disputa a Londres, Inglaterra el papel estelar en el establecimiento de las cotizaciones internacionales del oro, esta situación podría concretarse principalmente si China consigue la convertibilidad del yuan y con ello logra derribar el control hegemónico del patrón del dólar.

\section{5.- Continuar operaciones durante pandemia del COVID-19}

La pandemia del COVID-19 según Johns Hopkins University \& Medicine (2020), para el día 4 de octubre de 2020 a nivel mundial se habían registrado un total de 35,011,322 personas contagiadas y 1,034,865 fallecidas. En la región de América del Norte el COVID-19 ha tenido un fuerte impacto en la salud pública debido a que 8,339,314 personas han resultado contagiadas, equivalente al 23.8 por ciento del total de casos de contagios a nivel mundial. Mientras que las cifras de letalidad son aún más dramáticas porque han fallecido 298,161 personas, equivalente al 28.8 por ciento del total de personas que han perdido la vida a causa del COVID-19 a nivel mundial. Al revisar esos datos desglosados por cada uno de los tres países de América del Norte, observamos que se tenían registros de casos confirmados de contagios de 7,412,911 personas y 209,749 fallecidos (uno por cada 1,560 habitantes) en Estados Unidos de América, ubicado en primer lugar mundial en ambos rubros; en México son 757,953 personas contagiadas (noveno lugar mundial) y 78,880 fallecidos, uno por cada 1,581 habitantes (cuarto lugar mundial) y en Canadá 168,450 contagiados (26 lugar mundial) y 9,532 fallecidos, uno por cada 3,687 habitantes (20 lugar mundial). 
A pesar de esas cifras de graves afectaciones a la salud pública por la pandemia global y ante la necesidad de satisfacer la demanda creciente de minerales, varias economías del mundo continuaron con sus operaciones de exploración y explotación de minerales, como el caso de China con el cobre, carbón, hierro y el uranio. Durante el periodo de la pandemia del COVID19 este país ha continuado con su estrategia global de inversión de capitales destinados al acaparamiento de tierras y a proyectos de exploración minera, principalmente en regiones del sur global como América Latina y el África Subsahariana (World Energy Trade, 2020).

En la misma lógica que las empresas mineras de origen chino, también las empresas transnacionales que cuentan con sede corporativa en alguno de los tres países de América del Norte continuaron con sus operaciones, aunque en algunos casos suspendieron de forma temporal o parcial sus actividades, por lo general se mantuvo la actividad de exploración, explotación y beneficio de minerales durante la pandemia global del COVID-19. Dicha situación de acuerdo con MiningWatch (2020a: 5, 6), provocó diversos casos de contagios, como los 25 trabajadores que dieron positivo a COVID-19 en Canadá en la mina de paladio Lac des lles y ocho indígenas de comunidades aledañas a dicha mina; a los que se sumaron los 45 trabajadores contagiados en la mina Kearl Lake de Imperial Oil, así como el contagio que propagaron los trabajadores mineros en otras 159 personas. Así como los dos trabajadores mineros contagiados en la Unidad minera Tayahua de Grupo Frisco en México.

Esta situación para el caso mexicano según Uribe (2020), se presentó a lo largo del primer semestre del 2020, a pesar de las afectaciones a las comunidades mineras y de vulnerar el derecho humano a la salud, derivado de los contagios de COVID-19 a los trabajadores de las unidades de explotación de minerales (Movimiento M4, 2020).

Ante dichos problemas socioambientales ocasionados por la megaminería, surgieron también acciones colectivas de resistencia desde lo local, articuladas a través de 335 organizaciones nacionales e internacionales, quienes emitieron y difundieron un documento escrito, a través del cual manifestaron: "Solidaridad mundial con las comunidades, los pueblos indígenas y los trabajadores en peligro de ser víctimas de los especuladores de las pandemias mineras. [...] Las empresas mineras están ignorando la amenaza real de la pandemia y continúan sus operaciones". (MiningWatch, 2020b: 1, 24).

\section{6.- Violencia a defensores de los territorios con presencia minera.}

La crisis climática que padecemos a escala planetaria, está manifestándose en diversos problemas, conflictos y movimientos de justicia ambiental, los cuales, a su vez, han significado el agotamiento de los recursos naturales, la violación de los derechos humanos y en el peor de los casos, la pérdida de vidas humanas. Esto como una expresión donde las empresas de capital privado transnacional con el respaldo de los gobiernos nacionales están golpeando cada vez con mayor grado de violencia, a los pobladores de comunidades principalmente campesinas e indígenas que se resisten a los megaproyectos extractivos.

Un aspecto a destacar es que la numeralia de asesinatos de ambientalistas en el mundo, a nivel regional y sectorial, destaca que los defensores del territorio y los recursos naturales son asesinados principalmente en países del sur global y sectorialmente en actividades relacionadas con industrias extractivas y la agroindustria, (ver figuras 8 y 9 ).

Los datos de la figura 8, indican un incremento del 29.3 por ciento en el número de asesinatos de ambientalistas alrededor del mundo, al pasar de 164 durante el año 2018 a 212 durante el 
134 Extractivismos, conflictos mineros y desarrollo desigual en América del Norte

año 2019. Además, de los tres países de la región de América del Norte, únicamente en México se registraron casos de asesinatos de ambientalistas, con un incremento del 28.6 por ciento en el número de casos en los últimos dos años, porque durante el año 2018 alcanzó 14 personas asesinadas, ubicándose en el sexto lugar mundial y para el año 2019, la cifra creció a 18 personas asesinadas y el país se ubicó en el cuarto lugar mundial (Global Witness, 2019: 8 y 2020: 9).

Figura 8. Número total de ambientalistas asesinados por país, 2018 y 2019.

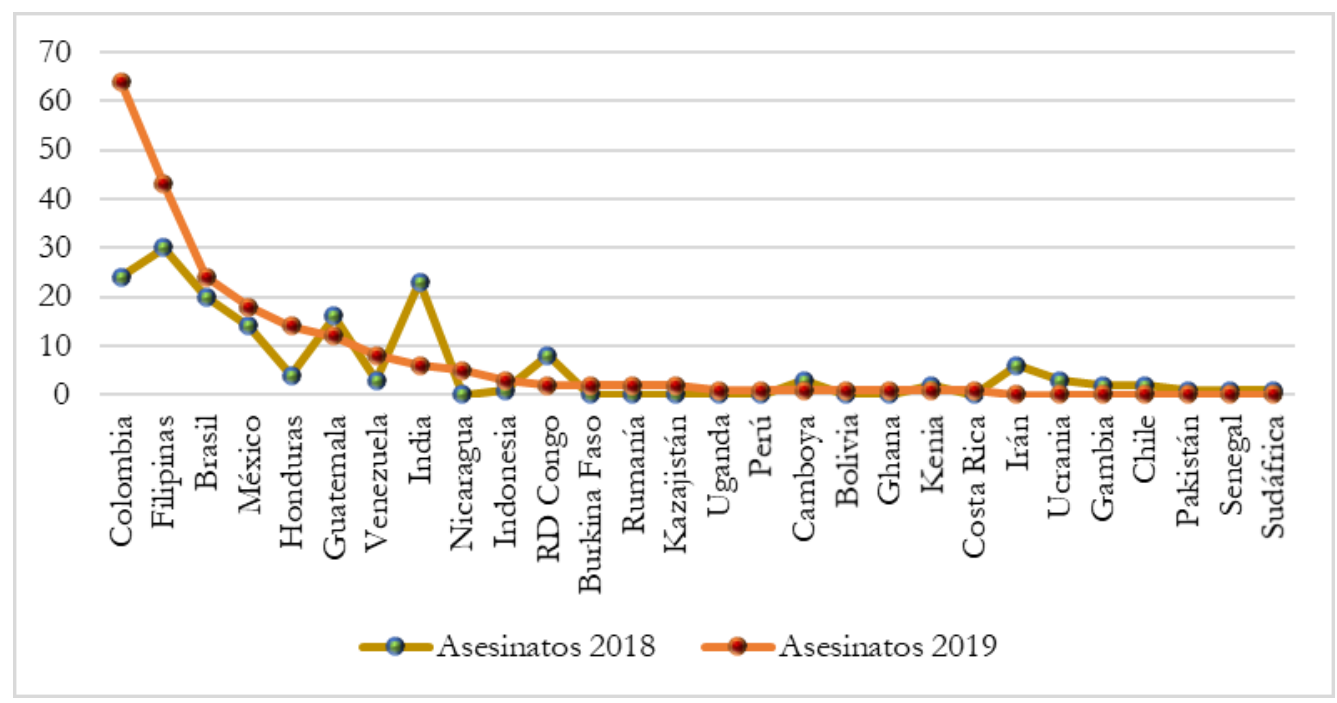

Fuente: Global Witness (2019: 8 y 2020: 9).

Figura 9. Número total de ambientalistas asesinados por sector, 2018 y 2019.

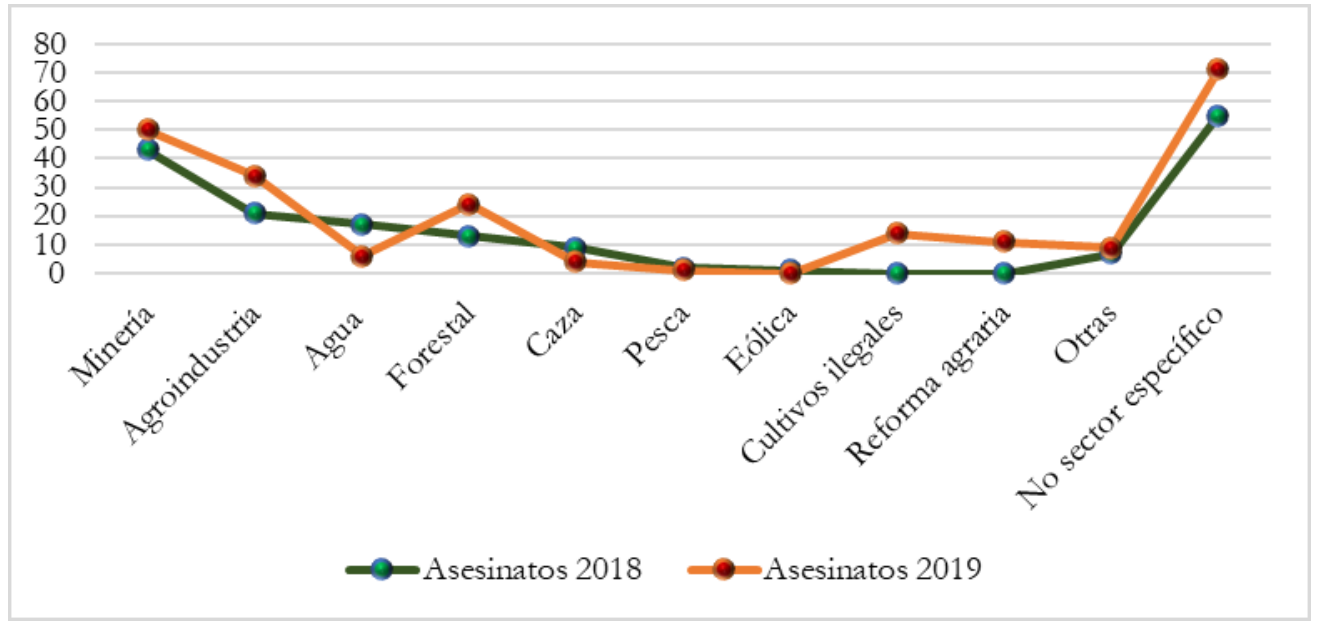

Fuente: Global Witness (2019: 8 y 2020:9).

Los datos de la figura anterior, indicaron que, de todos los rubros, la minería e industrias extractivas es el que mayor número de asesinatos de ambientalistas registró a nivel mundial. 
Además de que los asesinatos en este rubro se incrementaron un 16.3 por ciento en tan solo dos años, al pasar de 43 en el año 2018 a 50 durante el año 2019.

\section{7.- Donativos de Responsabilidad Social Empresarial}

La Responsabilidad Social Empresarial es uno de los dispositivos que poseen las empresas de capital privado transnacional, para posicionarse de manera positiva en las localidades donde operan y legitimar sus acciones para contar con aliados en las relaciones mina-comunidad.

Las empresas mineras líderes en producción de oro y plata a nivel mundial emprendieron diversas acciones de mitigación y apoyo a las comunidades en el marco de la pandemia del COVID-19, según datos de Newmont (2020), dicha empresa estadounidense, creó el Fondo de Apoyo Comunitario Global COVID-19, mediante el cual ha invertido más de USD\$9 millones de dólares en los rubros de salud pública, seguridad alimentaria y laboral. Por su parte en Barrick (2020), se destacó que la empresa minera transnacional de origen canadiense ha destinado más de USD $\$ 20$ millones de dólares de inversión social para la adquisición de equipo médico e insumos hospitalarios durante la emergencia sanitaria del COVID-19 en beneficio de la población de los países anfitriones donde opera.

Durante el año 2020 en el marco de la pandemia del COVID-19, según la Camimex (2020), las empresas mineras afiliadas a dicha organización otorgaron 2,280,183 insumos médicos, desde guantes, cubrebocas, gel antibacterial, batas y productos alimenticios, en beneficio de pobladores de 690 localidades de 22 entidades de la república mexicana. Entre los principales donantes fueron Peñoles que aportó un monto de $\$ 90$ millones de pesos, así como Grupo México que donó 450 mil kits médicos, 25 mil caretas y 125 mil pares de guantes, entre otros insumos hospitalarios.

\section{Conclusiones}

Los resultados del presente estudio corroboran nuestro argumento en el sentido de que la minería a gran escala en la región de América del Norte, ha sido uno de los factores clave que, junto con otros extractivismos, acompañados de diversos aspectos de orden económico, político y socioambiental, ha contribuido a favorecer la dinámica de desarrollo desigual entre los tres países que conforman esta región. Donde Canadá y Estados Unidos de América han sido los principales beneficiados de la renta minera y excedente de la extracción y beneficio de minerales metálicos. Mientras que en México el común denominador de 38 años de aplicación de políticas de corte neoliberal, le implicaron al país acumular más conflictos mineros y asesinatos por megaproyectos de muerte, convertido así en el territorio de sacrificio, que suministra fuerza de trabajo y materias primas para sostener el nivel de vida y bienestar de las sociedades canadiense y estadounidense.

En términos estructurales resultó relevante analizar lo que sucedió con el extractivismo minero en la región de América del Norte durante 38 años de la era neoliberal, donde la apertura comercial se formalizó mediante el TLCAN durante el año de 1994, luego renegociado en el año 2020 en la modalidad de T-MEC. Sobre todo, si tomamos en cuenta que la región de América del Norte, ocupa un lugar central en cuanto el poder económico y político a nivel mundial, aunque también acumula contradicciones sociales, de injusticia ambiental y de distribución de la riqueza. Además, en una mirada de conjunto concentra 16 de cada 100 kilómetros cuadrados de la superficie territorial de todos los países del mundo y cuenta también con 6 de cada 100 habitantes del planeta. En cuanto a la producción minera 
de oro América del Norte concentra cerca de 16 de cada 100 toneladas de la producción mundial del mineral aurífero y más de 28 de cada 100 toneladas de la plata que se produce a escala global.

La dinámica de desarrollo desigual en América del Norte, asociada a los extractivismos y conflictos mineros, se expresó en:

Diez de cada 100 de los 3,255 conflictos de justicia ambiental del mundo registrados al 30 de septiembre de 2020, se presentaron en la región de América del Norte, además Estados Unidos de América, México y Canadá se ubicaron en el cuarto, sexto y décimo segundo lugar mundial, respectivamente.

Diez de cada 100 de los 637 conflictos mineros del mundo, se concentraron en la región de América del Norte, además, México, Canadá y Estados Unidos de América, se ubicaron en el tercero, noveno y décimo primer lugar mundial, respectivamente.

$\mathrm{Al}$ establecer la proporción de conflictos mineros en relación al total de conflictos socioambientales por país en América del Norte, encontramos que en México la minería concentra 29 de cada 100 conflictos socioambientales, en Canadá fueron 23 de cada 100 y en Estados Unidos de América únicamente ocho de cada 100.

De los 63 conflictos mineros registrados en la región de América del Norte, cuatro de cada diez fueron de intensidad alta en México, cerca de uno de cada diez en Canadá y cerca de dos de cada diez en Estados Unidos de América, mientras que el promedio mundial es de tres de cada diez.

Las cifras anteriores muestran evidencia de que México se convirtió en la región de América del Norte en el territorio de sacrificio, con el mayor número de conflictos mineros y con el mayor grado de violencia y represión que aplican los corporativos mineros y los cuerpos policiacos de los tres órdenes de gobierno del Estado mexicano, para despojar de manera forzada a la población de su territorio y los recursos naturales.

La ampliación de las fronteras del extractivismo minero, gradualmente avanza hacia territorios de zonas semiurbanas y urbanas, aunque actualmente predomina en zonas rurales, y en esa dinámica el mayor afectado es México, porque en Estados Unidos de América nueve de cada 10 conflictos mineros ocurrieron en el medio rural, en Canadá ocho de cada diez y en México un poco más de seis de cada diez.

$\mathrm{Al}$ revisar los resultados de las acciones colectivas de resistencia local frente los megaproyectos de minería a gran escala, encontramos que, en Canadá, una cuarta parte tuvieron éxito de justicia ambiental, mientras que en México solo dos de cada diez casos y en Estados Unidos de América, únicamente cerca de uno de cada diez casos.

La contaminación del agua y destrucción de biodiversidad, concentraron cerca de cinco de cada diez de los casos de impactos ambientales ocasionados por la minería a gran escala en América del Norte.

Por su parte los elementos distintivos de las siete tendencias de la minería a gran escala en el marco de la crisis financiera de 2020, relacionada con los efectos del COVID-19, se expresaron en: 
1.- Un incremento del 423.3 por ciento en los precios internacionales del oro durante el periodo de 1982-2020, destacando un 35.4 por ciento de incremento durante los primeros nueve meses del año 2020, hasta alcanzar un precio máximo histórico de los USD \$2,067.15 dólares por onza del metal precioso.

2.- Un aumento del 4.3 por ciento en las reservas de oro en bancos centrales a nivel mundial durante el periodo de 2000-2020, hasta acumular las 34,891.5 toneladas de oro durante el año 2020, de los cuales, en América del Norte se concentraron cerca de 24 de cada 100 toneladas de las reservas de oro en bancos centrales.

3.- La relación desigual capital-trabajo en la minería en América del Norte, se expresó en que los trabajadores mineros de Canadá y Estados Unidos de América, reciben un salario 15 veces mayor por jornada diaria, que los trabajadores mineros en México.

4.- China disputa el imperialismo minero canadiense, al comprar activos de empresas mineras de Canadá, tiene el control de la producción y el mercado de los minerales para tecnologías renovables.

5.- La pandemia del COVID-19 en la región de América del Norte afectó a cerca de 24 de cada 100 de las personas contagiadas y 29 de cada 100 de las personas fallecidas a nivel mundial. Debido a que la minería fue clasificada como actividad esencial, ocasionó que se presentaran diversos casos de trabajadores mineros contagiados en las unidades mineras, además de la propagación del COVID-19 en perjuicio de pobladores de comunidades cercanas a las unidades mineras. Tal situación generó que al menos 335 organizaciones nacionales e internacionales alzaran la voz, ante la amenaza que representa la doble pandemia: el COVID-19 y la megaminería.

6.- México fue el único país de la región de América del Norte que registró casos de violencia durante el año 2019 por megaproyectos extractivos, al grado que concentró cerca de nueve de cada 100 de los casos de asesinatos de ambientalistas a nivel mundial.

7.- Las empresas mineras de capital privado transnacional en los tres países de América del Norte, crearon diversos fondos de apoyo comunitario, para atender la emergencia sanitaria del COVID-19, mediante donativos de equipo médico e insumos hospitalarios.

La situación de desarrollo desigual, ligada a los extractivismos y conflictos mineros en América del Norte, nos coloca en un desafío a escala regional, que involucra a las empresas mineras de capital privado, a los Estados nacionales, a las organizaciones de la sociedad civil defensoras de los recursos naturales, para que conjuntamente con los espacios del conocimiento científico, construyamos alternativas hacia otra forma de hacer minería.

\section{Referencias}

Banco Mundial (2019). Población total. Recuperado de https://datos.bancomundial.org/ indicator/SP.POP.TOTL

Banco Mundial (2020). Superficie (kilómetros cuadrados). Recuperado de https://datos.bancomundial.org/ indicator/AG.SRF.TOTL.K2

Barrick (2020). Una estructura sólida y una cultura de asociación impulsan una respuesta pronta y eficaz a una pandemia. Toronto, Canadá. Recuperado de https://www.barrick.com/English/about/covid19/default.aspx

Bejerano, P. (2019). Tierras raras: para qué sirve el dopaje de la electrónica. Recuperado de https://elpais.com/tecnologia/2018/12/18/actualidad/1545137160_888048.html 


\section{Extractivismos, conflictos mineros y desarrollo desigual en América del Norte}

Camimex (2020). Acciones de la industria minera nacional ante la emergencia por Covid-19. Ciudad de México, México. Recuperado de https://camimex.org.mx/files/2515/9560/0903/covid24julio.pdf

Centro de Información sobre Empresas y Derechos Humanos (2015). México: Proyecto de minería marina Don Diego, de Odyssey Marine Exploration, tendría impactos negativos en la pesca y el medio ambiente, según locales y ONG. EE.UU. Recuperado de https://www.business-humanrights.org/es/\%C3\%B Altimas-noticias $/ \mathrm{m} \% \mathrm{C} 3 \% \mathrm{~A} 9 \mathrm{xico}$-proyecto-de-miner $\% \mathrm{C} 3 \% \mathrm{ADa}$-marina-don-diego-de-odysseymarine-exploration-tendr $\% \mathrm{C} 3 \% \mathrm{ADa}$-impactos-negativos-en-la-pesca-y-el-medio-ambienteseg $\% \mathrm{C} 3 \%$ BAn-locales-y-ong/

Consejo Mundial del Oro (2020a). Producción de minas de oro. Recuperado de https://www.gold.org/ goldhub/data/historical-mine-production

Consejo Mundial del Oro (2020b). Precios del oro. Recuperado de https://www.gold.org/ goldhub/data/gold-prices

Consejo Mundial del Oro (2020c). Estadísticas de oferta y demanda de oro. Recuperado de https://www.gold.org/goldhub/data/historical-mine-production

Ejatlas (2020). Conflictos socioambientales en México. Recuperado de https://ejatlas.org/country/mexico

El Economista (2 de diciembre de 2019). Zijin Mining acuerda comprar a minera Continental Gold por 1,000 millones de dólares. El Economista. Recuperado de https://www.eleconomista.com.mx/empresas/ Zijin-Mining-acuerda-comprar-a-minera-Continental-Gold-por-1000-millones-de-dolares-201912020064.html

Eme Equis (7 de septiembre de 2020). Se estanca la minería en México en la era de la 4T. Recuperado de https://m-x.com.mx/al-dia/se-estanca-la-mineria-en-mexico-en-la-era-de-la-4t

Francisco (2020). Carta Encíclica Fratelli Tutti. Recuperado de https://www.vidanuevadigital.com/wpcontent/uploads/2020/10/FRATELLI-TUTTI_espanol.pdf

Fraser Institute (2019). Survey of Mining Companies 2019. Recuperado de https://www.fraserinstitute.org/ sites/default/files/annual-survey-of-mining-companies-2019.pdf

Global Witness (2019). ¿Enemigos del Estado? De cómo los gobiernos y las empresas silencian a las personas defensoras de la tierra y del medio ambiente. Recuperado de https://www.globalwitness.org/es / campaigns/environmental-activists/enemigos-del-estado/

Global Witness (2020). Defender el mañana. Recuperado de https://www.globalwitness.org/es/defendingtomorrow-es/

Gudynas, E. (2015). Extractivismos. Ecología, economía y política de un modo de entender el desarrollo y la Naturaleza. Cochabamba, Bolivia: Centro Latino Americano de Ecología Social (CLAES) y Centro de Documentación e Información Bolivia (CEDIB).

Johns Hopkins University \& Medicine (2020). COVID-19 Dashboard by the Center for Systems Science and Engineering (CSSE) at Johns Hopkins University (JHU). Recuperado de https://coronavirus. jhu.edu/map.html

Lampadia (29 de abril de 2016). El eje de la gobernanza global se inclina hacia el Asia. China se hace un sitio en la fijación del precio del oro. Recuperado de https://www.lampadia.com/analisis/economia/chinase-hace-un-sitio-en-la-fijacion-del-precio-del-oro/

LeGrand, C. (2006). Historias transnacionales: nuevas interpretaciones de los enclaves en América Latina. En Nómadas, Revista del Instituto de Estudios Sociales Contemporáneos de la Universidad Central. Bogotá, Colombia, Clacso, 25, 144-154. Recuperada de http://www.redalyc.org/articulo.oa?id= 105115224013

Martínez, J. (2005). El ecologismo de los pobres: conflictos ambientales y lenguajes de valoración. Barcelona, España: Icaria.

Miningwatch (2020a). Voces desde el territorio. Cómo la industria minera mundial se está beneficiando con la pandemia de Covid-19. Recuperado de https://miningwatch.ca/sites/default/files/voces_desde_el_ territorio_-_web.pdf

Miningwatch (2020b). Solidaridad global con comunidades, pueblos indígenas y trabajadores: en riesgo por la industria minera que busca aprovecharse de la pandemia. Recuperado de https://miningwatch.ca/ sites/ default/files/informe_de_coyuntura_mineria_y_covid-19.pdf

Movimiento M4 (2020). Más de 300 organizaciones expresan solidaridad con comunidades y trabajadores afectados por el temerario, manera de beneficiar de la pandemia del COVID-19 por parte de la industria minera. Recuperado de https://movimientom4.org/2020/06/mas-de-300-organizaciones-expresan- 
solidaridad-con-comunidades-y-trabajadores-afectados-por-el-temerario-manera-de-beneficiar-de-lapandemia-del-covid-19-por-parte-de-la-industria-minera/

Naciones Unidas (2020). Estado Miembro. Recuperado de https://www.un.org/es/memberstates/index.html

Navarro, A. (2014). Retos y perspectivas de la minería en México. Recuperado de https://archivo. estepais.com/site/2014/retos-y-perspectivas-de-la-mineria-en-mexico/

Newmont (2020). Sensibilizar sobre un problema oculto y destacar los servicios para las familias. Recuperado de https://www.newmont.com/blog-stories/blog-stories-details/2020/Newmont-Works-withCommunity-Partners-to-Support-Families-in-Need-Raise-Awareness-of-Services-and-Highlight-whatis-Often-a-Hidden-Problem/default.aspx

Oficina de Estadísticas Laborales en EE.UU. (2018). Empleo y salarios en las industrias mineras. Recuperado de https://www.bls.gov/opub/ted/2018/employment-and-wages-in-mining-industries.htm

Robbins, P. (2004). Political ecology: a critical introduction (critical introductions to geography). Arizona, United States of America: Malden, Blackwell.

Roy, B. (2018). Los conflictos ecológico-distributivos en la India a vista de pájaro. Ecología Política, 55:2431.

Secretaría de Economía (2020). Minería. El sector minero-metalúrgico en México contribuye con el 2.4 por ciento del Producto Interno Bruto nacional. Recuperado de https://www.gob.mx/se/acciones-yprogramas $/$ mineria\#: : :text $=\mathrm{M} \% \mathrm{C} 3 \% \mathrm{~A} 9 \mathrm{xico} \% 20 \mathrm{en} \% 20 \mathrm{el} \% 20$ mundo, $\% 2 \mathrm{C} \% 20 \mathrm{yeso} \% 2 \mathrm{C} \% 20 \mathrm{oro} \% 20$ $\mathrm{y} \% 20$ cobre

Servicio Geológico Mexicano (2019). Anuario Estadístico de la Minería Mexicana, 2018, Edición 2019. Recuperado de http://www.sgm.gob.mx/productos/pdf/Anuario_2018_Edicion_2019.pdf

The Mining Association of Canada (2019). The State of Canada's Mining Industry, Facts \& Figures 2019. Recuperado de https://mining.ca/wp-content/uploads/2020/01/FF-English-Web.pdf

The Silver Institute (2020). Top 20 producing countries. Recuperado de https://www.silverinstitute. org/mine-production/

Turdera, G. (2012). La polémica de la minería a cielo abierto: ¿desarrollo sustentable o espejitos de colores? Recuperado de http://www.unitedexplanations.org/2012/02/20/la-polemica-de-la-mineria-a-cieloabierto-desarrollo-sustentable-o-espejitos-de-colores/\#

Uribe, S.E. (2020). Covid-19 y minería en México. Recuperado de https://www.ocmal.org/covid-19-ymineria-en-mexico/

World Energy Trade (2020). China podría reinvertir en su industria minera. Recuperado de https://www.worldenergytrade.com/metales/mineria/china-podria-reinvertir-en-su-industria-minera 